\title{
Nouvelles du Comité central
}

\section{Lors de ses séances de février et de mars 2009, le Comité central de la FMH a ...}

... élu E. Gähler comme représentant de la FMH auprès de l'Académie suisse de médecine militaire et de catastrophe;

... décidé de mettre à l'ordre du jour de l'Assemblée des délégués du 16 mars 2009 la question du tarif des émoluments versés par les non-membres;

... approuvé l'introduction de l'instrument que constitue le postulat tant pour l'Assemblée des délégués que pour la Chambre médicale; le postulat a pour but de discuter et d'élaborer des idées dignes d'intérêt, mais qui ne seraient pas encore acceptables dans une formulation directe;

... discuté le budget 2010 avec la Commission de gestion et approuvé ce budget à l'intention de l'Assemblée des délégués et de la Chambre médicale;

... reçu des informations sur la fondation Caisse de secours des médecins suisses;

... accepté de prendre en charge les frais de la réunion consultative des sociétés de médecine d'Autriche, d'Allemagne, de la Principauté du Liechtenstein, du Tyrol du Sud, du Luxembourg et de Suisse; cette réunion aura lieu à Zurich les 3 et 4 juillet 2009;

... accepté les propositions suivantes de l'association faîtière IPSILON (Initiative pour la prévention du suicide en Suisse) en vue du symposium «Suicide» prévu en mars 2010:

- participation de la FMH à ce symposium en tant qu'organisation partenaire,

- mise à disposition du logo de la FMH pour les documents publicitaires du symposium,

- soutien financier par la FMH;

... pris connaissance de l'activité du groupe de travail Palliative Care composé de représentants de l'Office fédéral de la santé publique ainsi que de l'Office fédéral de la formation professionnelle et de la technologie; ce groupe de travail clarifie quelles sont les offres de formation existantes ou à créer en la matière;

... accepté la proposition de porter à l'ordre du jour de la Chambre médicale du 28 mai 2009 l'intégration, dans le Code de déontologie de la FMH (Annexe 1), de la directive intitulée «Décisions de réanimation» adoptée par le Sénat de l'ASSM en novembre 2008;

... adopté les prises de position relatives:

- au projet de directives de l'ASSM sur l'établissement de directives anticipées du patient, moyennant la prise en compte complémentaire de l'étude pratique «PAVE»,
- au document de l'ASSM intitulé «Standards pour la publication de données relatives à la qualité du traitement médical»,

- à la consultation sur la «modification de l'ordonnance sur la protection contre le rayonnement non ionisant (ORNI)» et

- à la révision de la loi sur l'asile en ce qui concerne l'assistance médicale apportée aux requérants d'asile;

... chargé le Service juridique de proposer à l'Assemblée des délégués sa formulation de l'art. 55b LAMal relatif au rôle des cantons en matière de remise des autorisations de pratiquer;

... décidé de participer activement à la mise en œuvre de l'article 22a LAMal relatif à l'obligation de remettre, aux autorités fédérales compétentes, les données des fournisseurs de prestations;

... approuvé la suite du projet de monitorage de la qualité, selon les propositions du concept relatif à ce projet;

... pris connaissance du fait

- qu'à sa demande, la FMH a été élue membre du Nationaler Verein zur Förderung der Qualitätsentwicklung (Association nationale pour le développement de la qualité; NVQ) avec le statut $\mathrm{d}^{\prime}$ «observatrice»; le NVQ résulte de la fusion entre l'Association intercantonale pour l'assurance de la qualité dans les hôpitaux (AIQ) et le Service national de coordination et d'information pour l'assurance-qualité dans les établissements de santé (CIQ),

- que le projet soutenu par la FMH et intitulé «Elaboration d'un cadre d'action pour une approche systématique et constructive des incidents au sein des établissements» de la Fondation pour la sécurité des patients était entré dans sa phase de formation;

... reçu des informations sur l'organisation et le mode de travail de l'Institut suisse pour la formation médicale postgraduée et continue (ISFM), actif depuis le $1^{\text {er }}$ avril 2009;

... été renseigné sur les dossiers suivants du département Tarifs et conventions:

- Contrôle fédéral des finances,

- réponse et remise à la CdG du Conseil national d'un volumineux catalogue de questions relatives à la révision de la Liste des analyses;

... approuvé la version mise à jour de la prise de position de la FMH sur SwissDRG. 